\title{
CLÍNICA MICCIONAL COMO SÍNTOMA POR CUERPO EXTRAÑO INTRAVESICAL
}

Pablo Luis Guzmán Martínez-Valls, Beatriz Honrubia Vilchez, Almudena Rodríguez Tardido, Emilio Izquierdo Morejón, Bogdan-Nicolau Pietricica, Antonio Rosino Sanchez, Gregorio Hita Villaplana, Antonio Romero Hoyuela y Bernardino Miñana López.

Servicio de Urología. Hospital General Universitario Morales Meseguer. Murcia. España.

Resumen.- OBJETIVO: La presencia de cuerpos extraños intravesicales aparecen excepcionalmente, no siendo una urgencia habitual. La mayoría de cuerpos extraños son de origen erótico-sexual aunque no podemos olvidar otros como el abandono en el campo quirúrgico de algún elemento utilizado. A propósito de ello, revisamos la literatura.

MÉTODOS: Realizamos una búsqueda bibliográfica electrónica PubMed (MEDLINE) con términos MESH "Foreign-Body Migration" [MeSH] AND "Urinary Bladder" [MeSH] y de citas bibliográficas. Hacemos una revisión de la literatura estableciendo una clasificación atendiendo al origen y diagnóstico así como tratamiento. La gran mayoría de las publicaciones co- rresponden a aportación de casos nuevos. Describimos la forma de presentación más habitual que coincidía con el que tuvimos en nuestro Centro.

RESULTADOS: Encontramos un total de 122 trabajos de los cuales nueve correspondían a una revisión; seleccionamos en total 20. Aunque la mayoría son aportación de casos, las revisiones, establece una clasificación en función del origen: así, hacen referencia a los introducidos directamente a la vejiga: -por la propia persona: horquillas, imperdibles, lápices, hilos de cobre, pelos; -accidentales: balas; -iatrógenos: fragmentos de sonda o catéteres, grapas, suturas. Migrados desde otros lugares: de origen urológicos, ginecológico, digestivo, vascular. Estableceremos un algoritmo diagnóstico y terapéutico.

CONCLUSIONES: Los cuerpos extraños dentro de la vejiga no son tan infrecuentes como se cree. El hallazgo casual es lo más frecuentes. Los síntomas urinarios son los mas frecuentes y el antecedente de manipulación propia o de otros en esa esfera genito urológica y el tratamiento es la extracción utilizando el menos cruento y mas sencillo para el paciente.

Palabras clave: Cuerpos extraños intravesicales.

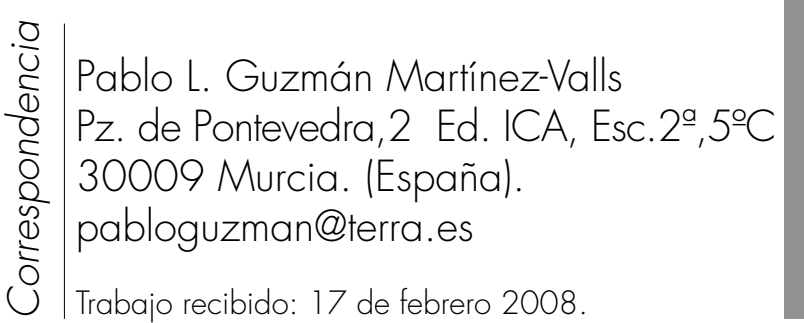

Summary.- OBJECTIVES: The presence of intravesical foreign bodies is exceptional; it is not a common emergency. Most foreign bodies have a sexual-erotic origin, although we cannot forget others such as elements left by the surgeon in the surgical field. We performed a bibliographic review on the topic.

METHODS: We performed a PubMed (MEDLINE) electronic bibliographic search with the mesh terms "fo- 
reign-body migration" [MESH] AND "Urinary Bladder" [MESH] and bibliographic citations. We performed a bibliographic review establishing a classification depending on the origin and diagnosis, as well as treatment. Most publications are case reports. We described the most frequent presentation which was the same than we have in our Center.

RESULTS: We found a total of 122 works, nine of which were reviews; we selected 20 works. Although most are case reports, the reviews establish a classification depending on the origin: so, they refer to those foreign bodies directly introduced into the bladder: 1. By the patient: hairpins, safety pins, pencils, copper wires, hairs; 2. Accidentally: bullets; 3. latrogenic: fragments of bladder or ureteral catheters, staples, sutures. Foreign bodies migrated from other places: urological, gynecological, gastrointestinal, or vascular origin. We established a diagnostic and therapeutic algorithm.

CONCLUSIONS: Bladder foreign bodies are not as frequent as it is believed. Most are found incidentally. Lower urinary tract symptoms are the most frequent, as the antecedent of manipulation by the patient or others in the genital-urologic sphere. The treatment of choice is extraction using the least invasive and most simple method for the patient.

Keywords: Intravesical foreign bodies.

\section{INTRODUCCIÓN}

La presencia de cuerpos extraños en el árbol urológico inferior no es tan infrecuente como podríamos imaginar $(1,2,3)$. La variedad de cuerpos extraños dentro o fuera del tracto genitourinario incluye todo tipo de objetos y resulta interesante porque en función del tipo así será su tratamiento. La revisión más extensa localizada es la realizada por Van Ophoven que revisa más de 800 casos durante los últimos doscientos cincuenta años y los divide en función del órgano implicado: pene, uretra, vejiga, ureter y riñón (4).

El motivo mas frecuente y común asociado a cuerpos extraños en el tracto urinario inferior es erótico-sexual, aunque existen otras causas como los migrados de otros lugares: mallas en hernias (5), dispositivos intrauterinos $(6,7)$, prótesis vasculares $(8)$, mallas de incontinencia, prótesis de pene, esfínteres artificiales,... La mayoría de los casos ocurren, cuando es por la primera causa, en pacientes con limitaciones psicológicas o accidentes masturbatorios.
Las manifestaciones clínicas mas frecuentes son síntomas urinarios como infecciones de repetición, litiasis vesical, polaquiuria, dolor y escozor miccional, hematuria; menos frecuentes son fístulas: vesicointestinal, vesicovaginal, vesicoacetabular (9) y alteración de función renal.

En muchas ocasiones se descubre como hallazgo casual al realizar una radiografía simple o ecografía. Tras la historia clínica, partiendo de una sospecha y tras una exploración genitourinaria se puede llegar al diagnóstico.

A raíz de la presentación de un caso que a continuación describimos, revisamos la literatura, estableciendo una clasificación atendiendo a su origen y un algoritmo diagnóstico y terapéutico.

\section{CASO CLÍNICO}

Paciente mujer de 74 años remitida desde Consulta Externa de Ginecología a Urología por Infecciones Urinarias (ITUs) de repetición con dolor hipogástrico de al menos seis meses de evolución.

Dentro de sus antecedentes personales destaca histerectomía vía vaginal por prolapso uterino

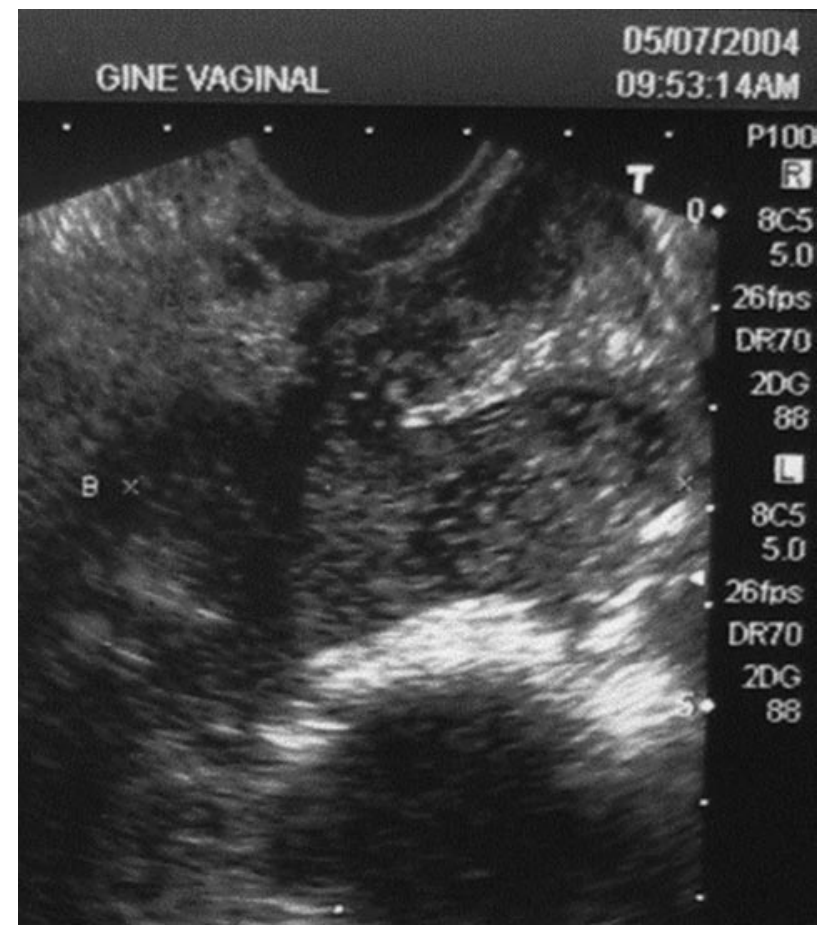

FIGURA 1. ECO ginecológica a los 18 días de la histerectomía. Sospecha de absceso de cúpula vaginal. 


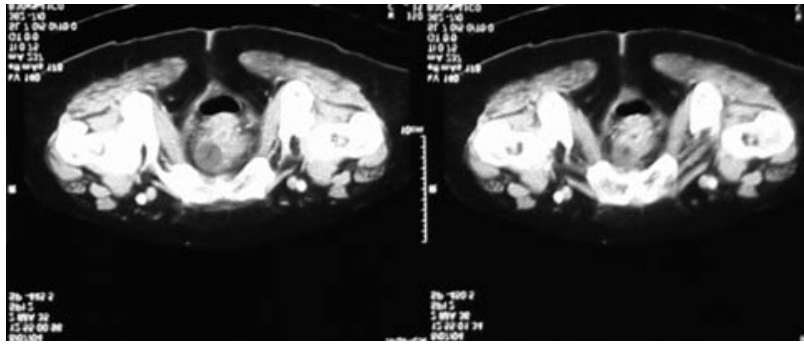

FIGURA 2. TC pélvico a los 18 días de la histerectomía. Absceso de cúpula vaginal.

hacía siete meses en otro centro, precisó reingreso a los 18 días de la cirugía por fiebre y dolor, con diagnóstico de absceso de cúpula vaginal que drena espontáneamente, desapareciendo el cuadro clínico; las pruebas que le practicaron en dicho ingreso muestran: ECO (Figura 1) y TC (Figura 2) absceso en cúpula vaginal sin ningún otro hallazgo. No DM. No HTA. No alergias conocidas. No hematuria. No cólicos. Infecciones de orina esporádicas. G2 AOV2, no escapes.

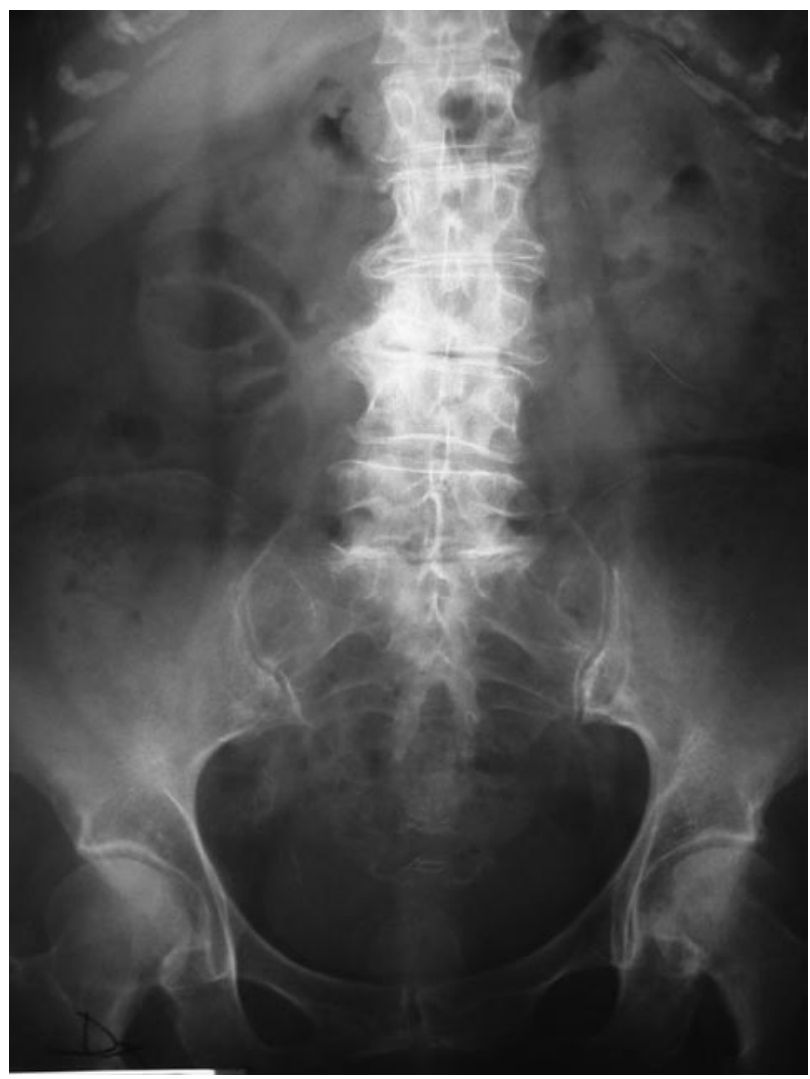

FIGURA 3. Rx. Aparato urinario: informada como normal. Se aprecia imagen débilmente cálcica en pelvis, en área declive.
Cuando es remitida a nuestra consulta por la clínica miccional e ITU, acompaña Rx aparato urinario simple (Figura 3) que es informada como normal, aunque se aprecia claramente contorno débilmente cálcico en pelvis en área vesical, y ecografía transvaginal que informa de probable litiasis vesical (Figura 4), diagnóstico por el cual junto a las infecciones de orina de repetición es remitido al Servicio de Urología de nuestro centro.

Ante el hallazgo en la Rx. simple que acompaña y la ecografía transvaginal con litiasis intravesical, no móvil y con el antecedente quirúrgico referido, se sospecha desde el inicio la presencia de cuerpo extraño intravesical por probable pérdida de material utilizado en la cirugía.

Se realiza uretrocistoscopia el mismo día de la consulta para confirmar sospecha diagnóstica que así se produce, apreciándose un gran "pelotón de gasas" adherida a la pared vesical que no se moviliza por estar calcificada; se solicita TC pélvico (Figura 5) para valorar extensión y tamaño así como las relaciones con el área.

Con el diagnóstico definitivo de litiasis intravesical por cuerpo extraño, abandonado probablemente en la cirugía de la histerectomía se propone extracción de la misma mediante cistoslitectomía o mas correctamente "cistogasomectomía" mediante apertura vesical a cielo abierto encontrando el cuerpo extraño descrito (Figura 6).

Tras buena evolución la paciente está asintomática, han desaparecido las infecciones de orina

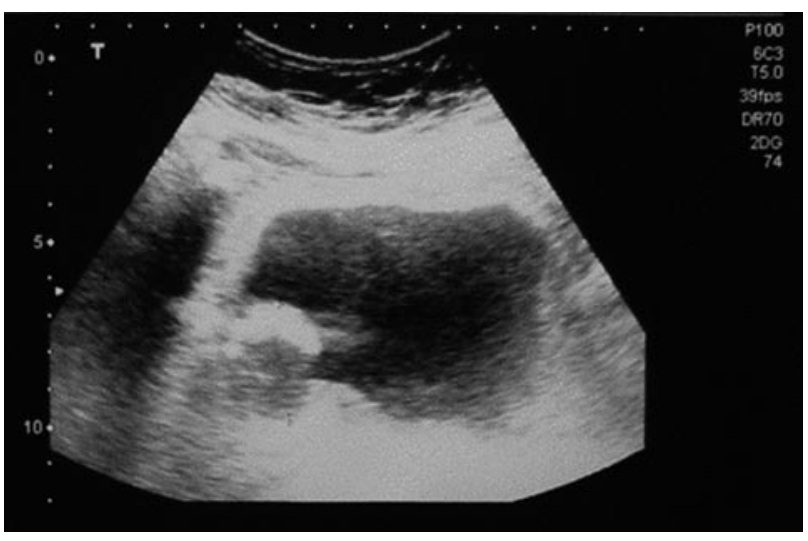

FIGURA 4. ECO ginecológica trasnvaginal: imagen cálcica en pared lateral vesical compatible con litiasis no móvil. 


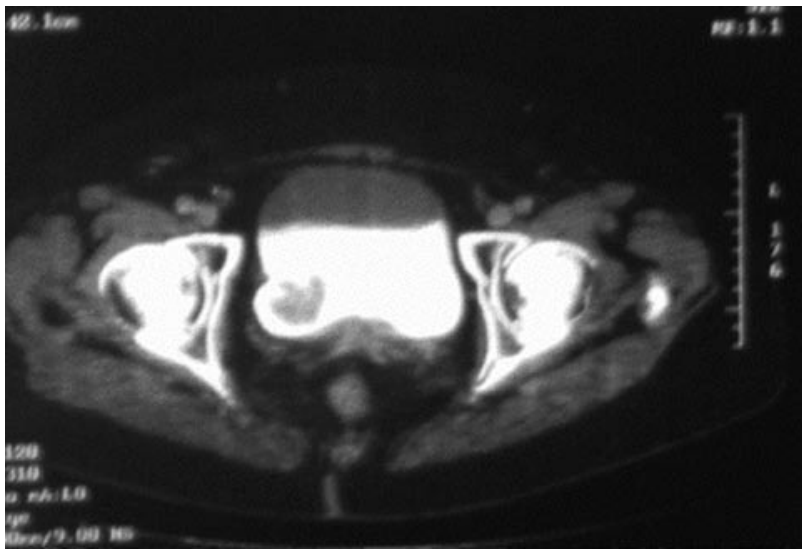

FIGURA 5. TC pélvico: cuerpo extraño calcificado dependiente de fondo derecho vesical, compatible con cuerpo extraño.

de repetición, aunque en los dos años de seguimiento ha tenido un episodio aislado, la paciente realiza una vida absolutamente normal.

\section{DISCUSIÓN}

El primer caso de cuerpo extraño externo publicado fue sobre el pene en 1755 (4) y se publican diferentes casos con diversos y variopintos tipos de objetos en todo el árbol urológico, éstos van desde anillos, nueces, férulas, botellas hasta bolígrafos, vegetales, hilos de cobre, clips, palos de caramelo, pasando por pequeñas serpientes y otros animales, con fines diversos $(1,4)$.

En función del origen del cuerpo extraño podemos establecer una clasificación $(1,3)$.

\section{Introducidos directamente a la vejiga}

- Por la propia persona: horquillas, imperdibles, lápices, minas, monedas, cables de luz, tornillos, clavos, huesos, hilo de pescar, pelos, palos de chupachups...

- Accidentalmente: balas...

- Latrógenos: dobles J, fragmentos de sonda, de catéteres, fragmentos de material endoscópico, suturas, grapas.

\section{Migrados desde otros lugares}

- De origen urológico: prótesis de incontinencia, partes de prótesis de pene, grapas quirúrgicas, puntos,...; - De origen ginecológico: DIUs, clips, pesarios,

- De origen digestivo: ingerido y fistulizado como espina de pescado, palillo de dientes, cuchilla de afeitar; material quirúrgico como grapas, mallas de hernias, gasas, prótesis digestiva.

- De origen vascular: prótesis vasculares.

Es importante también, conocer las características del paciente: asociación con alteraciones mentales, con fines erótico-masturbatorios o autolesivos o bien como consecuencia de una cirugía o actuación médica y por tanto no influye en nada el comportamiento del paciente.

Según la localización del cuerpo extraño así serán las manifestaciones clínicas (1-9). La más frecuente es la presencia de síntomas urinarios como las infecciones de repetición resistentes a antibióticos habituales, polaquiuria, disuria, escozor miccional, hematuria y litiasis; menos habituales son la presencia de fístulas con la presencia de neumaturia, fecularia o fugas de orina o expulsión espontáneas del cuerpo extraño (6-9). Muy poco frecuente es la presencia de insuficiencia renal de causa obstructiva.
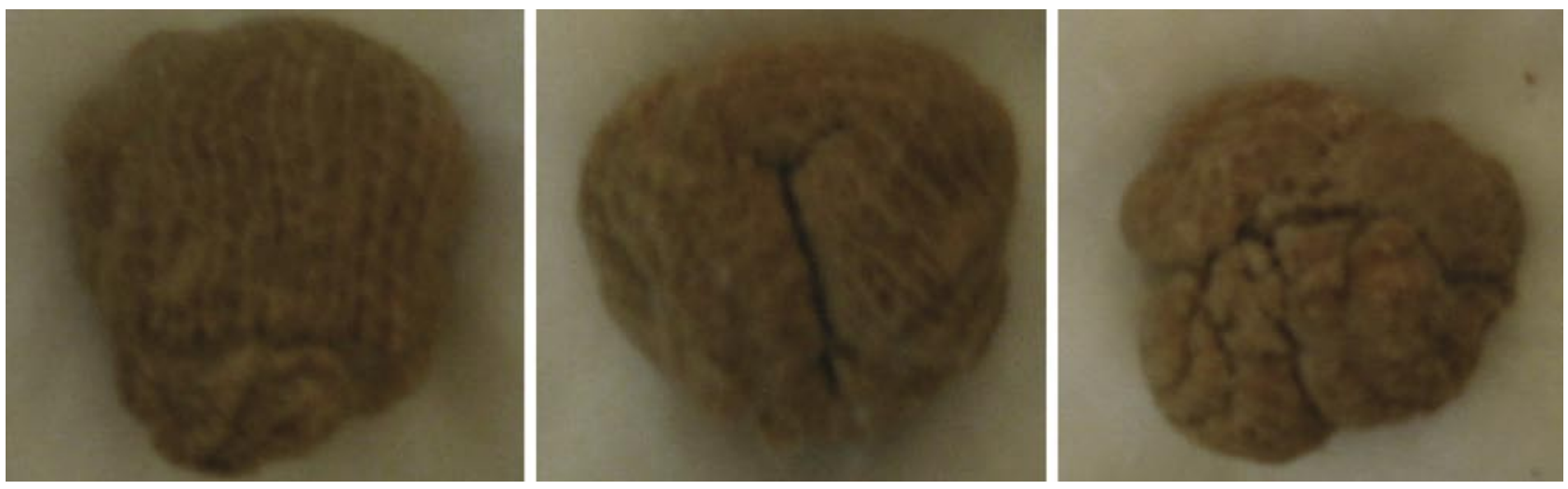

FIGURA 6. Cistogasectomía, pelotón de gasas calcificada. 
En ocasiones el diagnóstico es incidental ante la realización de una prueba de imagen como es una radiografía simple o una ecografía abdominal por otro motivo, ya que muchos pacientes, los que se han introducido voluntariamente un objeto, no reconocen mayoritariamente esa práctica.; en otras ocasiones se debe al origen insospechado por proceder de migraciones de otras localizaciones. Existen incluso extracciones de cuerpos extraños que han sido "perdidos/abandonados" más de 25 años. Cuando existe reconocimiento por maniobras masturbatorias o antecedentes quirúrgicos recientes con clínica miccional la sospecha puede ser elevada y las pruebas mas utilizadas para el diagnóstico serán la radiografía convencional, ecografia y uretrocistoscopia, siendo el TC y la RMN mas infrecuente.

El tratamiento dependerá de la localización del cuerpo extraño, tamaño, forma, movilidad, sexo, edad del paciente y lesiones asociadas. Los métodos mas frecuentes utilizados comienzan con el que menos morbilidad ocasione, y esto es la uretrocistoscopia, sola o asociándola a mínima incisión, cistostomia o mediante técnicas combinadas (1-9).

\section{CONCLUSIONES}

Por tanto y a modo de conclusiones destacamos que los cuerpos extraños dentro de la vejiga no son tan infrecuentes como se puede pensar. El hallazgo casual es lo más frecuente al realizar alguna prueba por otro motivo.

Los síntomas más frecuentes son los urinarios: polaquiuria, dolor miccional, escozor miccional, hematuria, infecciones de repetición. El antecedente de "automanipulación" o por "otros" en la esfera urológica: instrumentación, prótesis, tratamientos quirúrgicos..., pueden ser los responsables de la presencia de cuerpos extraños en la vejiga.

Las pruebas diagnósticas mas frecuentes y resolutivas suelen ser la radiografía simple, la ecografía y la uretrocistoscopia. Los métodos utilizados para la extracción de cuerpos extraños vesicales son la uretrocistoscopia, sóla o en combinación con otras. Es muy importante tener en cuenta el tratamiento de las posibles complicaciones asociadas: fístulas, lesiones uretrales, alteraciones psicológicas, etc.

\section{BIBLIOGRAFÍA y LECTURAS \\ RECOMENDADAS ( ${ }^{*}$ lectura de interés $y$ ** lectura fundamental)}

**1. BOSQUET, M.; GIMENO ARGENTE, V.; PALMERO, J.L. y cols.: "Cuerpos extraños uretrovesicales: Nuestra experiencia". Actas Urol. Esp., 29: 572, 2005.

*2. BRIONES, G.; JIMÉNEZ, M.; SAEZ, J. y cols.: "Cuerpos extraños uretrovesicales". Actas Urol. Esp., 25: 456, 2001.

**3. PASCUAL, D.; GARCÍA DE JALÓN, A.; MALLÉN, E. y cols.: "Cuerpos extraños intravesicales. Revisión de la Literatura". Actas Urol. Esp., 27: 265-2003.

**4. VAN OPHOVEN, A.; DEKERNION, B.: "Clinical Management of Foreign bodies of the genitourinary tract". J. Urol., 164: 274, 2000.

*5. KURUKAHVECIOGLU, O.; EGE, B.; YAZICIOGLU, O. y cols.: "Polytetrafluoroethulene prosthesis migration into the bladder after laparoscopic hernia repair: A case report". Surg. Laparosc. Endosc. Percutan. Tech., 17: 474, 2007.

*6. CECCATO, V.; BOILEAU, A.; ROBLIN, M. y cols.: "Migration of an intra uterine device into the bladder". Prog. Urol., 17: 256, 2007.

*7. CORONEL, B.; SANCHEZ, M.J.; CARRASCOSA, V. y cols.: "Migration of an intrauterine contraceptive device into the urinary bladder: Repot of one case". Arch. Esp. Urol., 57: 75, 2004.

*8. HÄCKER, A.; UYSAL, Z.; LANGBEIN, S. y cols.: "Foreign body in the urinary bladder after vascular surgery". Aktuelle Urol., 36: 249, 2005.

*9. WILHELM, S.; SCHWÄBLEIN, T.; BUCHNER. y cols.: "Spontaneous vesicoacetabular cuatenous fistula after total hip replacement". Urologe A., 46: 163, 2007. 